\title{
Inhomogeneous broadening and spectral diffusion of the CdSe nanocrystals in the solid nanocomposites
}

\author{
Konstantin Magaryan ${ }^{1,2, *}$ \\ ${ }^{1}$ Moscow State Pedagogical University, 119991 Moscow, Russia \\ ${ }^{2}$ Institute for Spectroscopy RAS, 108840, Moscow, Russia
}

\begin{abstract}
The mechanisms of broadening of optical spectra of CdSe nanocrystals (quantum dots, QD) synthesized in the liquid crystalline matrix were studied. The experiment confirms that the luminescence spectra of macroscopic samples are broadened inhomogeneously. In addition, small fractions of nanocomposites (with single QDs and small ensembles of QDs) manifest noticeable stochastic spectral diffusion.
\end{abstract}

The object under study is nanocomposite with CdSe nanocrystals (quantum dots, QD). Nanocrystals were grown in the ionic liquid crystalline matrix of cadmium alkanoate using template synthesis [1]. It's quite a new method of synthesis QDs inside liquid crystalline host medium. The resulting object combines peculiarities of semiconductor heterostructures and the liquid crystal anisotropic medium. In emission spectra of ensemble [2, 3] samples under study show two separated bands in vicinity of exciton transition and wide band in the long wavelength region (see Fig. 1a). In this talk we report on luminescence spectra of small fractions of such nanocomposites, which include single QDs and/or small ensembles of QDs, as well as about comparative analysis with the spectra of macroscopic samples.

Site-selective spectroscopy is a very powerful tool for detailed study of dynamics in a doped disordered solids $[4,5]$. The luminescence and absorption (fluorescence excitation) spectra for the samples were obtained using luminescence microscopy technique and reported in $[2,3]$. The luminescence spectra of small fractions of the sample (isolated micro- and nanoclusters which contains single QD or their small ensembles) were obtained with the help of setup for a spatially resolved single molecule detection system based on the fluorescent microscope $[4,6]$ with the home-built prism spectrometer. The samples were prepared by spin-coating of low concentrated colloidal toluene solutions with nanocomposites powder. The luminescence was excited by $405 \mathrm{~nm} \mathrm{cw}-$ laser. Luminescence signal was collected in confocal scheme from isolated nano-/micro-particles.

The luminescence spectra from different particles (Fig. 1b) demonstrate strong inhomogeneous broadening of the macroscopic sample. On the figure 1 (c) several examples of the recorded photoluminescence spectra of the same diffraction-limited area (some selected isolated particle) are shown. Effect is observed under continuous cw-laser excitation at room temperature. Such random jumps in the emission wavelength called spectral diffusion lead to the broadening of the nanocrystals ensemble luminescence

\footnotetext{
* Corresponding author: ka.magaryan@mpgu.edu
} 
spectra. Luminescence from QDs with different size going along with phenomenon of spectral diffusion leads to inhomogeneous broadening of the spectra of the composite.
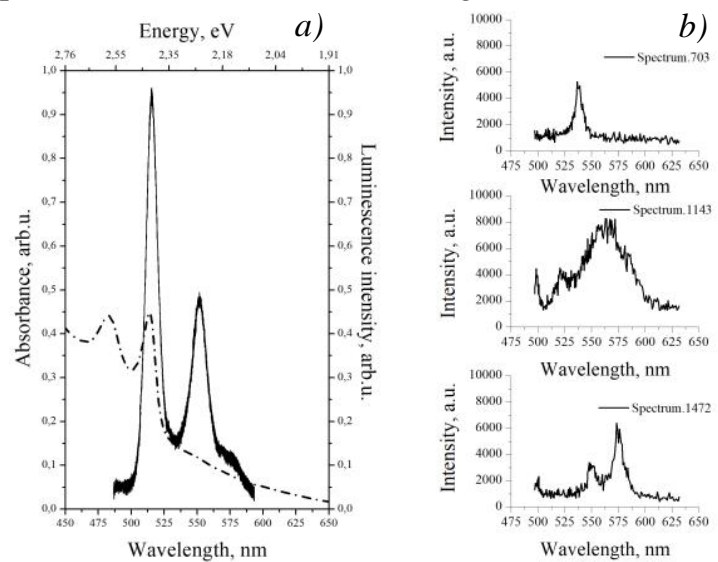

b)

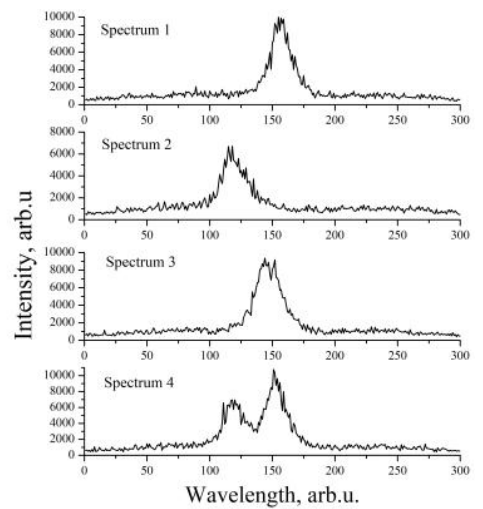

Fig. 1. Optical spectra of the investigated nanocomposites with CdSe QDs $(2.3 \mathrm{~nm})$. (a) The macroscopic sample. (b) Three different isolated small $\mathrm{nm} / \mu \mathrm{m}$-sized fractions of the sample. (c) The same nm-sized particle (with near-single QD) as measured sequentially in different time moments.

There is a possibility to analyze relation of such spectral diffusion process to single QD blinking process [7-10]. In fact, the fluorescence trace for single QDs can be build. We observe the random duration of the onloff-intervals. When several fluorescence tracks are summarized, the effect of fluorescence blinking is no longer observed. Events fluctuations are complex with different amplitudes. If we propose that there are several emitting particles, then the probability of their simultaneous switch to the off-state would be low.

The photon distribution function for a single QD can be reconstructed from a fluorescence trace. As we have found for many cases the complicated fluorescence intermittency pattern cannot be explained in the context of the Efros-Rosen charge model [7]. In our case long on-intervals are absent and the fluorescence fluctuates between multiple states with different intensity. We describe such blinking in the general framework of the two models: multiple recombination center model [8] or combined model based on the modified charging model and the two-level system model $[9,10]$. This dynamics can be described using second approach that takes into account ionization/neutralization processes in a nanocrystal and fluctuations of atoms on a QD interface.

This work was supported by RFBR (proj. no 18-02-01121 - study of spectral diffusion in a new luminescent nanomaterials) and Scientific Council of the President of the Russian Federation (proj. no. MK-342.2017.2 - study of the broadening of the nanocomposites spectra). The data for analysis was obtained in collaboration with Dr. I.Yu. Eremchev and Prof. A.V. Naumov. The author is grateful to them as well as to Prof. I.A. Vasilieva for the fruitful discussions.

\section{References}

1. D. Zhulai et al., NRL, 10, 66 (2015)

2. K.A. Magaryan et al., J. Lumin., 169, 799 (2016)

3. K.A. Magarian et al., J. Phys: Conf. Ser., 478, 012007 (2013)

4. A.V. Naumov, Physics Uspekhi, 56, 605 (2013)

5. K.R. Karimullin, A.V. Naumov, J. Luminescence, 152, 15 (2014)

6. A.V. Naumov, I.Yu. Eremchev, A.A. Gorshelev, Eur. Phys. J. D, 68, 348 (2014)

7. A.L. Efros, M. Rosen, Phys. Rev. Lett., 78, 1110 (1997)

8. P.A. Frantsuzov, S. Volkan-Kacso, B. Janko, Phys. Rev. Lett., 103, 207402 (2009)

9. I.S. Osad'ko, I.Y. Eremchev, A.V. Naumov, J. Phys. Chem. C, 119, 22646 (2015)

10. I.Y. Eremchev, I.S. Osad'ko, A.V. Naumov, J. Phys. Chem. C, 120, 22004 (2016) 